\title{
Analysis of Coherent MPSK-OFDMA Signals in FSO Communication System under Complex Weather Conditions
}

\author{
Junyi Zhou ${ }^{1}$, Yufeng Shao ${ }^{1, a}$, Yue Zhou ${ }^{1}$, Changxiang Li $^{1}$, Zhifeng Wang ${ }^{1}$, Wenzhe Ma ${ }^{1}$, \\ XingPing $\mathrm{Ji}^{1}$ and Jianjun Wang ${ }^{1}$ \\ ${ }^{1}$ Faculty of Engineering, Shanghai Polytechnic University, Shanghai, China \\ asyufeng@163.com
}

Keywords: MPSK modulation, CO-OFDMA, FSO, Complex weather.

\begin{abstract}
Free space optical (FSO) communication is a potential technology for last-mile applications. The major advantages are the high transmission rates, inherent security, and unlicensed spectrum. However, in practical applications, the main challenge for FSO communication systems is the performance degradation imposed by the different atmospheric conditions. In this paper, coherent optical orthogonal frequency division multiplexing access (CO-OFDMA) technique is adopted in the FSO communication system. $\mathrm{M}$-ary phase shift keying (MPSK, $\mathrm{M}=4,8,16$ ) downstream signals are transmitted at $40 \mathrm{~Gb} / \mathrm{s}$ for 100 meters and 300 meters transmission under five weather conditions (sunny, light rain, moderate rain, snow, heavy fog). And the characteristics of the received signals are analyzed. In sunny day, the received phase information of constellation diagram is obvious, and the BER is $10^{-4.5}$. In rainy, snow and foggy days, the system can still maintain communication for short transmission.
\end{abstract}

\section{Introduction}

In the era of ever-present connectivity, the requirement of high speed networks had put the pressure on wireless communication networks. Free Space Optics (FSO) networks become a significant building block for wide area space networks and a promising solution for the last mile problem, due to its key advantages including ease of deployment, license-free operation, high transmission security, high bit rates[1-2]. However, FSO requires line of sight (LOS) propagation and transceiver alignment to establish a wireless optical link. But complex weather conditions such as rain, snow, and fog poses threat to this LOS. Optical signals will suffer from absorption, scattering etc, thus the range and the capacity of wireless channel are degraded[3,4]. Orthogonal frequency division multiplexing access (OFDMA) has become a leading multiple access candidate scheme for beyond fourth/fifth generation $(4 \mathrm{G} / 5 \mathrm{G})$ wireless systems, due to its flexibility in resource allocation and robustness against multi-path fading effect[5-9]. In this paper, we employ the coherent modulation technique to improve the receiver sensitivity, the effects of absorption and scattering to 
MPSK-OFDMA signals can be reflected by the free space channel attenuation coefficient. The performance of FSO communication system under different weather conditions is analyzed.

\section{MPSK-OFDMA-FSO Communication System}

As shown in Fig. 1, MPSK-OFDMA-FSO system can be divided into five parts: OFDMA modulation/demodulation module, Optical-electrical conversion module and FSO channel. The main function of the OFDMA modulation module is to map the original bit stream into $\mathrm{N}$ parallel sub-channels by serial/parallel conversion (S/P), and different sub-carriers are assigned to different modulation pattern as shown in Fig. 2. For the OFDMA demodulation module, its function is the opposite of the procedure described above. The main function of the electric/optical conversion module is to convert the OFDMA signals which is on the electric frequency into the optical domain by the external modulation of the optical I/Q modulator. The optical I/Q modulator consists of a pair of parallel arms Mach-Zehnder Modulators (MZMs). The electrical signal is split into two channels, which are led to the two ports of the I/Q modulator respectively. Electric field amplifier (EA) is used to make the phase shift of the lower arm input signal is $90^{\circ}$. At the optical/electric conversion module, optical signals and the local light are interfered by the optical field through the mixer. The signal phase difference of the input balanced dual-detector is made $90^{\circ}$ by the phase shifter to detect the in-phase component and the quadrature component of the OFDMA signals. Finally, four PIN PD are used to convert the OFDMA optical signals into an electrical signal.

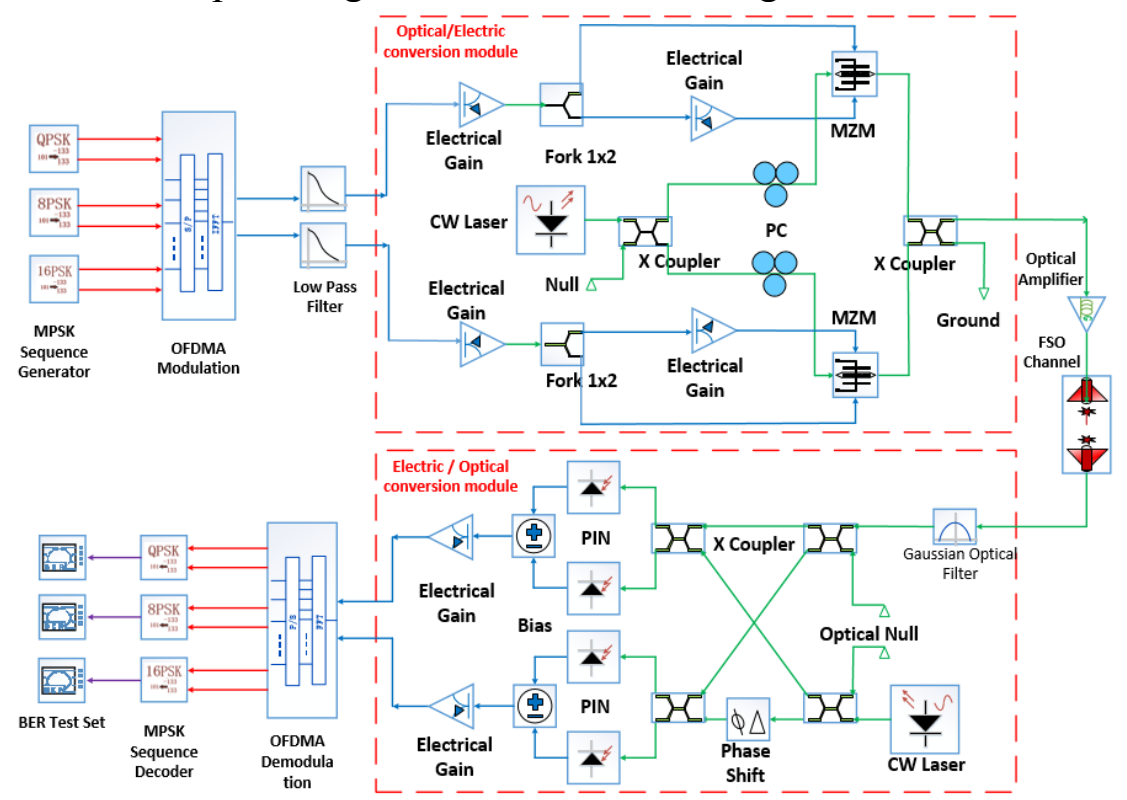

Fig.1. MPSK-OFDMA-FSO system

The FSO channel module is composed of a transmitting telescope, free space and a receiving telescope. The attenuation of laser power is mainly determined by three main parameters: atmospheric attenuation, geometric loss, transmission distance between transmitting and receiving telescope. In the sunny, light rain, moderate rain, snow, fog weather conditions. Atmospheric attenuation value are shown in Fig. 3 below. 


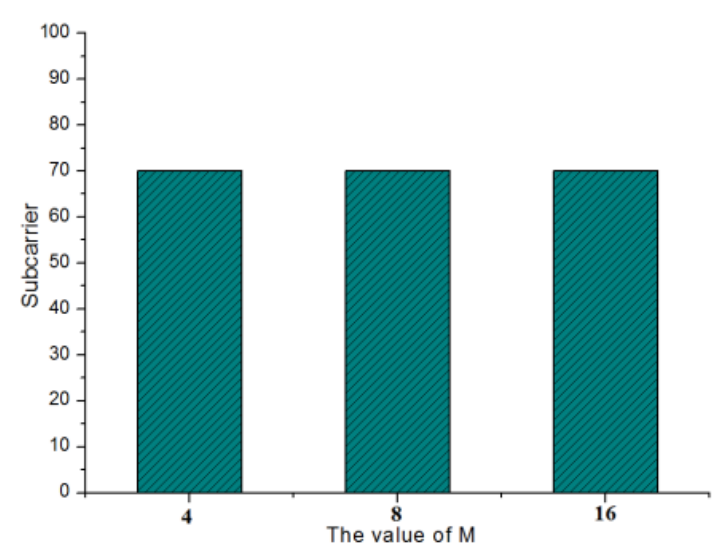

Fig. 2. Sub-carrier allocation

\begin{tabular}{|c|c|}
\hline Weather & $\begin{array}{c}\text { Free space channel } \\
\text { attenuation }\end{array}$ \\
\hline Sunny & $3 \mathrm{db} / \mathrm{km}-7 \mathrm{db} / \mathrm{km}$ \\
\hline Light rain & $7 \mathrm{db} / \mathrm{km}-15 \mathrm{db} / \mathrm{km}$ \\
\hline Moderate rain & $25 \mathrm{db} / \mathrm{km}-40 \mathrm{db} / \mathrm{km}$ \\
\hline Snow & $100 \mathrm{db} / \mathrm{km}-150 \mathrm{db} / \mathrm{km}$ \\
\hline Fog & $200 \mathrm{db} / \mathrm{km}-300 \mathrm{db} / \mathrm{km}$ \\
\hline
\end{tabular}

Fig. 3. Transmission losses under 5 typical weather conditions

\section{Results Analysis}

Using the MPSK-OFDMA-FSO system, several tests were carried out under the typical outdoor weather conditions, and the statistical analysis of the measured simulation data was carried out. The OFDMA optical signal is transmitted through the atmospheric channel for 100 meters and 300 meters, the receiving power and spectrum is shown in Fig. 3 and Fig. 4. At the receiving end, the signal power is affected by weather conditions and the peak power of the optical spectrum decreases gradually in Fig. 3 and Fig. 4.

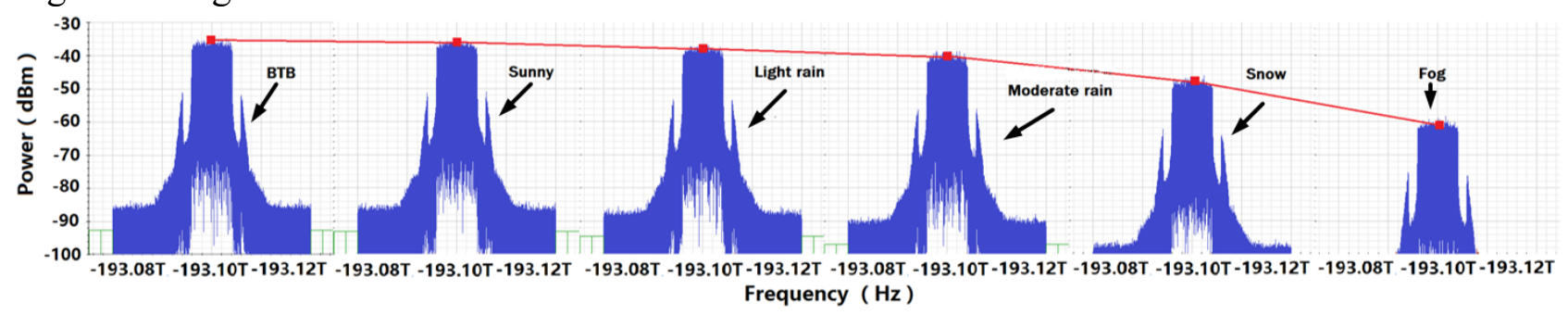

Fig. 3. Optical spectrum diagrams of the received signals transmitted 100 meters under different weather conditions

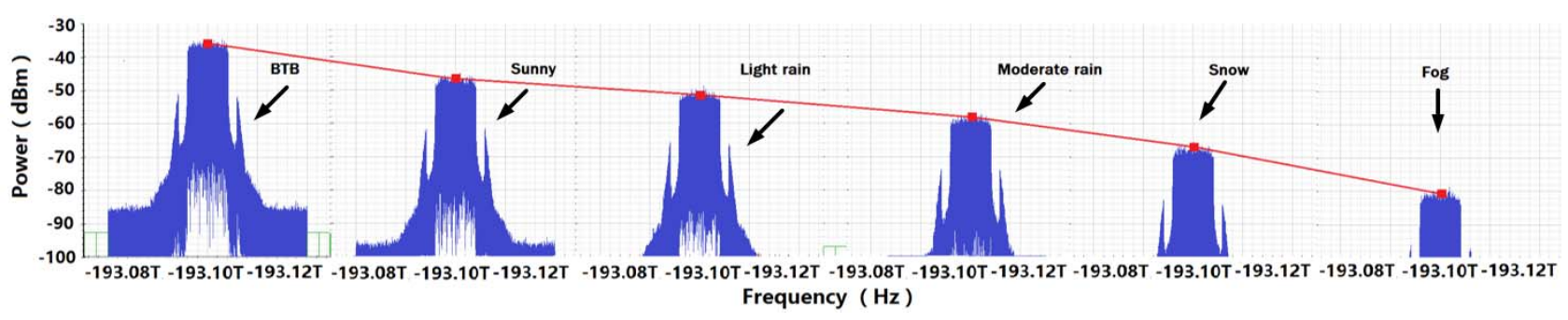

Fig. 4. Optical spectrum diagrams of the received signals transmitted 300 meters under different weather conditions 

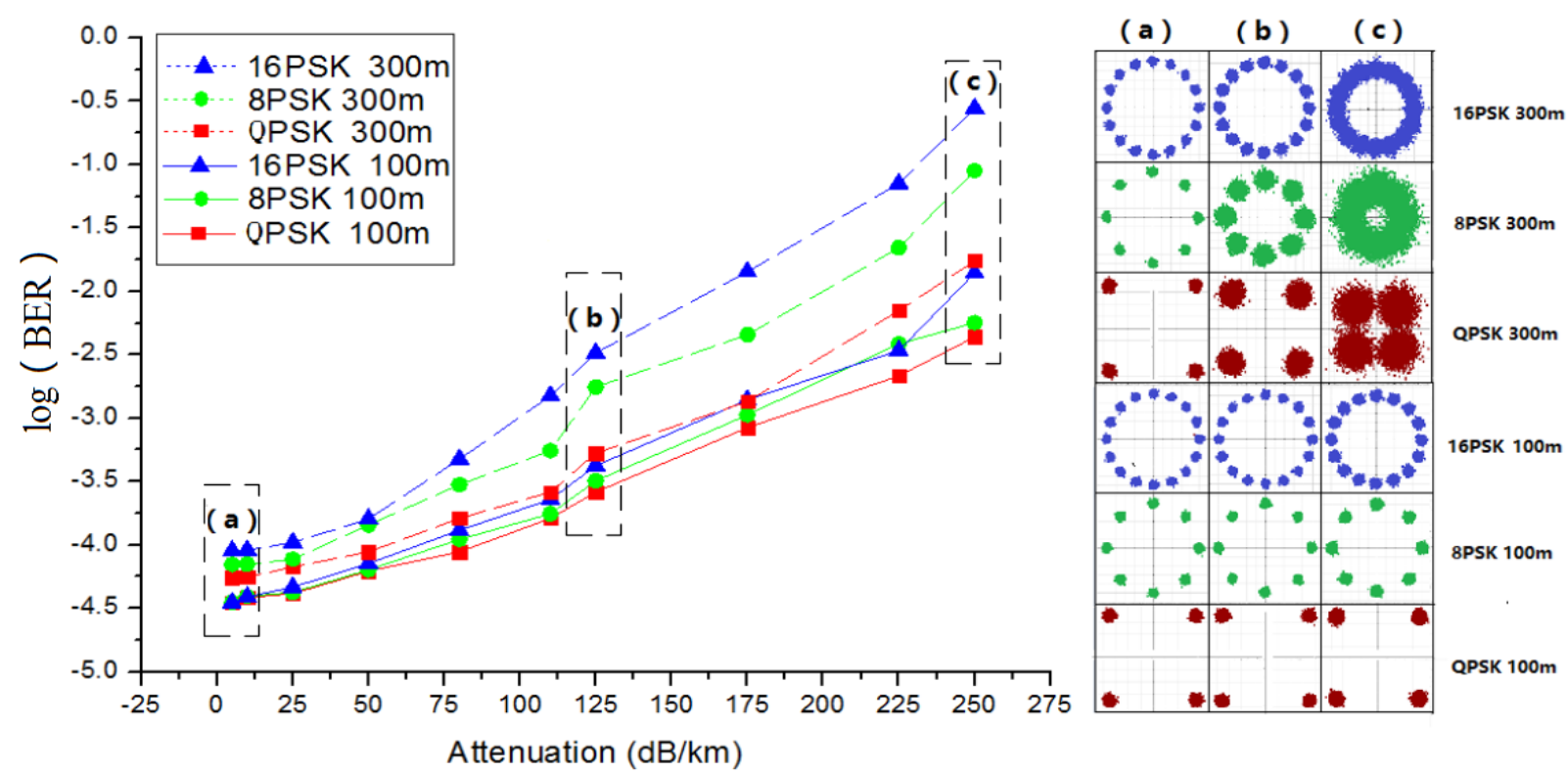

Fig. 5. The relationship between free space channel attenuation and BER at distance of $100 \mathrm{~m}$ and $300 \mathrm{~m}$

The influence of sunny weather on the transmission quality is the least, and the influence of light rain, moderate rain, snow and fog transmission quality is larger and larger. The free space channel attenuation value is very small $(5 \mathrm{~dB} / \mathrm{km})$ in sunny days. As shown in Fig. 5(a), the constellation diagram points are arranged neatly in the 2-dimensional space, the constellation diagram point has no aliasing and the distribution focus is clear.The density of water mist and rain drops is bigger in rainy days, which makes the energy of some light beams scatter around. So it's easy to understand that: No matter how far the transmission distance is, moderate rain attenuates the phase and the amplitude of the optical signal more strongly than Light rain, as shown in Fig. 5(b). Fig. 5(c) shows the constellation diagram of the signals at the transmission distance is $100 \mathrm{~m}$ and $300 \mathrm{~m}$ under fog weather. The effect of fog on the signal attenuation is extremely serious in long distance. The constellation shows the diffusion, distortion, deformation of the vector points. At the transmission distance is $300 \mathrm{~m}$, the constellation diagram is presented as a fuzzy block or ring, we can not distinguish the phase and amplitude information. It is worth noting that, as shown in Fig. 5(c) rear part, although the weather is fog, the transmission distance is only short as 100 meters, the constellation diagram is still visible.

\section{Summary}

In this paper, a novel $40 \mathrm{~Gb} / \mathrm{S}$ MPSK-OFDMA-FSO system is designed and analyzed. A short-distance communication link is introduced under different weather conditions and transmission distances. With the deterioration of the weather environment, the dispersion of receiving constellation diagram becomes more and more serious and the phase information becomes blurred. As we can see, the transmission performance of QPSK-OFDMA signals under different weather conditions is always better than the other cases, and this implementation is simple and reliable. However, 16PSK-OFDMA signals have higher transmission efficiency, and its modulation method has higher complexity and cost budget. Moreover, its transmission reliability is the worst. 8PSK-OFDMA as a compromise of the modulation scheme, it may be applied in FSO systems in the future. 


\section{Acknowledgments}

This work was financially supported by the National Natural Science Foundation of China (No.61107064), Innovation Program of Shanghai Municipal Education Commission (No.15ZZ101), Leading Academic Discipline Project of Information and Communication Engineering (No.XXKZD1605), School Foundation (No.EGD14XQD01) of Shanghai Polytechnic University, College Students' Science and Technology Innovation Project of Shanghai Polytechnic University (No.2016-xjkj-063), Connotation construction project of Shanghai Polytechnic University (No.A11NH170301).

\section{References}

[1]. Shao Y, Chi N, Fan J, et al. Generation of 16-QAM-OFDM Signals Using Selected Mapping Method and Its Application in Optical Millimeter-Wave Access System[J]. IEEE Photonics Technology Letters, 2012, 24(15):1301-1303.

[2]. Shao Y, Wang Y, Chi N. 60-GHz RoF system with low PAPR 16QAM-OFDM downlink using PTS segmentation[J]. IEEE Photonics Technology Letters, 2013, 25(9):855-858.

[3]. Rajendrakumar S M, Karruppaswamy M. Analysis of link availability in FSO-OFDM system under various climatic conditions[J]. Engineering Journal, 2015, 19(1).

[4]. Zhou J, Shao Y, Wang Z, et al. A 16PSK-OFDM-FSO Communication System under Complex Weather Conditions[J]. 2016, 06(8):131-135.

[5]. Zhang M, Liu Y. Energy Harvesting for Physical-Layer Security in OFDMA Networks[J]. IEEE Transactions on Information Forensics \& Security, 2016, 11(1):154-162.

[6]. Venturino L, Zappone A, Risi C, et al. Energy-Efficient Scheduling and Power Allocation in Downlink OFDMA Networks With Base Station Coordination[J]. IEEE Transactions on Wireless Communications, 2015, 14(1):1-14.

[7]. Wang Y, Wang D, Ma J. On the Performance of Coherent OFDM Systems in Free-Space Optical Communications[J]. IEEE Photonics Journal, 2015, 7(4):1-10.

[8]. Hao Yaohong, Wang Rong, Li Yuquan, et al. Investigation of Polarization Effect in Coherent Optical Orthogonal Frequency Division Multiplexing System[J]. Acta Optica Sinica, 2013, 33(7):122-127.

[9]. Wang Y, Wang D, Ma J. On the Performance of Coherent OFDM Systems in Free-Space Optical Communications[J]. IEEE Photonics Journal, 2015, 7(4):1-10. 